\title{
A promising concept of combination therapy for positional obstructive sleep apnea
}

\author{
Marijke Dieltjens • Anneclaire V. Vroegop • Annelies E. Verbruggen • \\ Kristien Wouters • Marc Willemen • Wilfried A. De Backer • Johan A. Verbraecken • \\ Paul H. Van de Heyning • Marc J. Braem • Nico de Vries • Olivier M. Vanderveken
}

Received: 15 July 2014 /Revised: 30 September 2014 / Accepted: 7 October 2014 /Published online: 22 October 2014

(C) Springer-Verlag Berlin Heidelberg 2014

\begin{abstract}
Purpose The objective of this randomized controlled trial was to assess the additional effect of a chest-worn sleep position trainer (SPT) in patients with residual supine-dependent obstructive sleep apnea (sdOSA) under mandibular advancement device (MAD) therapy.

Methods Baseline and follow-up polysomnography with MAD were performed. Twenty patients with sdOSA under
\end{abstract}

Trial registration: Clinicaltrials.gov: NCT01535521

M. Dieltjens $(\bowtie) \cdot$ M. J. Braem
Department of Special Care Dentistry, Antwerp University Hospital
(UZA), Wilrijkstraat 10, 2650 Edegem, Belgium
e-mail: Marijke.Dieltjens@uza.be

M. Dieltjens $\cdot$ A. V. Vroegop · A. E. Verbruggen ·

P. H. Van de Heyning • N. de Vries • O. M. Vanderveken

ENT Department and Head and Neck Surgery, Antwerp University

Hospital (UZA), Edegem, Belgium

K. Wouters

Scientific Coordination and Biostatistics, Antwerp University

Hospital (UZA), Edegem, Belgium

M. Willemen · W. A. De Backer J J. A. Verbraecken •

P. H. Van de Heyning • O. M. Vanderveken

Multidisciplinary Sleep Disorders Centre, Antwerp University

Hospital (UZA), Edegem, Belgium

W. A. De Backer · J. A. Verbraecken

Pulmonary Medicine, Antwerp University Hospital (UZA), Edegem, Belgium

M. Dieltjens · A. V. Vroegop - A. E. Verbruggen - W. A. De Backer • J. A. Verbraecken • P. H. Van de Heyning - M. J. Braem •

N. de Vries $\cdot$ O. M. Vanderveken

Faculty of Medicine and Health Sciences, University of Antwerp,

Antwerp, Belgium

N. de Vries

Department of Otolaryngology/Head and Neck Surgery, Sint Lucas

Andreas Hospital, Amsterdam, Netherlands
MAD therapy underwent two consecutive randomized polysomnographies: one with SPT and one with combination of SPT + MAD. Data are presented as median (quartile 1, quartile 3).

Results The SPT reduced the time spent in supine sleeping position compared to baseline and MAD therapy. Both MAD and SPT were individually effective in reducing the overall apnea/hypopnea index (AHI) significantly when compared to baseline from $20.8(15.1 ; 33.6) / \mathrm{h}$ at baseline to $11.0(6.7$; $13.8) / \mathrm{h}$ and to $11.1(3.5 ; 17.7) / \mathrm{h}$ with MAD or SPT, respectively. The combination of SPT + MAD further reduced the overall AHI to $5.7(3.6 ; 7.4)$, which was significantly lower than with MAD alone $(p<0.001)$ and SPT alone $(p<0.008)$, respectively.

Conclusions The results of this study indicate that combination of SPT + MAD leads to a higher therapeutic efficacy in patients with sdOSA under MAD therapy when compared to one of the treatment modalities alone.

Keywords Obstructive sleep apnea/hypopnea syndrome . Sleep-disordered breathing $\cdot$ Supine-dependent

\section{Introduction}

Approximately $50-60 \%$ of patients with obstructive sleep apnea (OSA) suffer from supine-dependent OSA (sdOSA) defined according to Cartwright [1] as having twice as many respiratory events in the supine sleeping position compared to the non-supine sleeping position. Mador et al. [2] defined sdOSA as having twice as many respiratory events in the supine position than in non-supine positions along with an apnea/hypopnea index (AHI) $<5$ events/ $h$ in the non-supine position and a 15-min threshold for sleep in supine and nonsupine position. A third definition of sdOSA was introduced 
by Marklund et al. [3, 4] defined as a supine $\mathrm{AHI} \geq 10$ events/h together with a non-supine AHI of $<10$ events $/ \mathrm{h}$.

Positional therapy is a treatment modality aimed at preventing sleep in the supine position [5]. It is recently shown during drug-induced sleep endoscopy (DISE) that a change of body position from supine to lateral position leads to improvement of upper airway collapse in patients with sdOSA [6]. In the future, DISE could be performed in both the supine and lateral positions [7]. The most widely used technique to avoid the supine sleeping position involves strapping a bulky object to the back of the patient thereby preventing supine positioning. Several studies have shown that such therapies have a significant positive effect on snoring and OSA severity in patients with sdOSA [5, 8-12]. However, the bulky object is uncomfortable for patients and results in disturbed sleep and low long-term compliance rates [8,9]. Therefore, positional therapy has not found its way into daily OSA treatment routine to date [13]. In order to overcome such compliance problems, both a new neck-worn device and a chest-worn device correcting the supine sleeping position by activating a vibration alarm were evaluated. This novel concept of positional therapy showed promising results in reducing apnea severity, together with a higher compliance $[14,15]$.

Oral appliance therapy is a non-invasive treatment for patients with snoring and OSA [16]. The most common type of oral appliances prescribed for the treatment of OSA is the mandibular advancement device (MAD) worn intraorally at night in order to reduce upper airway collapse by protruding the mandible [4, 17-19]. This treatment modality, although not as effective as continuous positive airway pressure (CPAP) in reducing apnea severity, is preferred by the majority of patients and has a relatively high objective compliance rate [20-22].

In a recent study, it was found that up to $34 \%$ of patients treated with MAD therapy showed sdOSA under MAD therapy [23]. Our research hypothesis is that those patients could benefit from additional positional therapy.

The aim of this prospective randomized controlled trial (RCT) was to investigate the feasibility of the additional use of a chest-worn sleep position trainer (SPT) in patients with sdOSA under MAD therapy.

\section{Material and methods}

Participants, setting, and study design

In Belgium, CPAP is the standard treatment for patients with moderate to severe OSA (AHI $>20$ events/h) and is fully reimbursed in these patients. Therefore, our policy is to propose a trial of CPAP therapy to any patient with an AHI of at least 20 events/h. In our multidisciplinary setting, patients with mild OSA (AHI $<20$ events/h) and patients with CPAP intolerance are referred to the multidisciplinary dental sleep clinic involved in the decision-making for the implementation of MAD therapy [24].

Twenty patients with a residual sdOSA (AHI $\geq 5 / \mathrm{h}$ ) following both Cartwright's [5] and Marklund's [3, 4] criteria under MAD therapy were randomly selected out of our patient population. At the start of MAD therapy, patients were not judged suitable for MAD treatment if they suffered from any pre-existing active temporomandibular joint dysfunction, if their dental status or periodontal health precluded them from wearing an oral appliance, or if they were fully edentulous $[25$, 26]. In addition, patients with other sleep disturbances than sleep-related breathing disorders were excluded from the study.

The patients were invited to participate in this RCT design in which two treatment modalities were tested in a randomized order: the SPT and combination of SPT + MAD therapy. The other inclusion criteria were $\geq 20 \%$ of total sleep time spent in the supine position during polysomnography (PSG) with $\mathrm{MAD}$ and unchanged body mass index $\left(\mathrm{BMI} \pm 1 \mathrm{~kg} / \mathrm{m}^{2}\right)$ between diagnostic PSG and inclusion in the study.

Seven patients had a baseline AHI $<20$ events/h and were therefore CPAP naive. CPAP therapy was offered in the other 13 patients: 10 of them could not tolerate the CPAP device, two patients refused to start CPAP therapy, and one patient wanted an alternative for CPAP. The study design included a baseline level I PSG and a follow-up PSG with the MAD in situ. These PSGs were performed in routine clinical practice to assess the therapeutic efficacy of the MAD.

Patients were randomly allocated in a 1:1 ratio to be treated with either the SPT prior to combination therapy or combination therapy prior to the SPT, see Fig. 1. At the end of the study, but before knowing the results of the consecutive study nights during the RCT, patients were asked to fill out a questionnaire about their personal therapy preference (MAD, SPT, or combination of SPT + MAD).

The reported trial was conducted in accordance with the institutional guidelines of the ethical committee (Belgian registration number: B300201212947) and informed consent was obtained. The trial was registered at clinicaltrials.gov (NCT01535521).

\section{Oral appliance}

Patients were treated with a custom-made, titratable MAD (Respident Butterfly, Dormoco, Nijlen, Belgium $(n=14)$ or SomnoDent Flex, Somnomed AG, Australia $(n=6))$. After receiving full explanation of the titration procedure, patients were instructed to gradually titrate the MAD until subjective 
Fig. 1 Flow chart of the study design

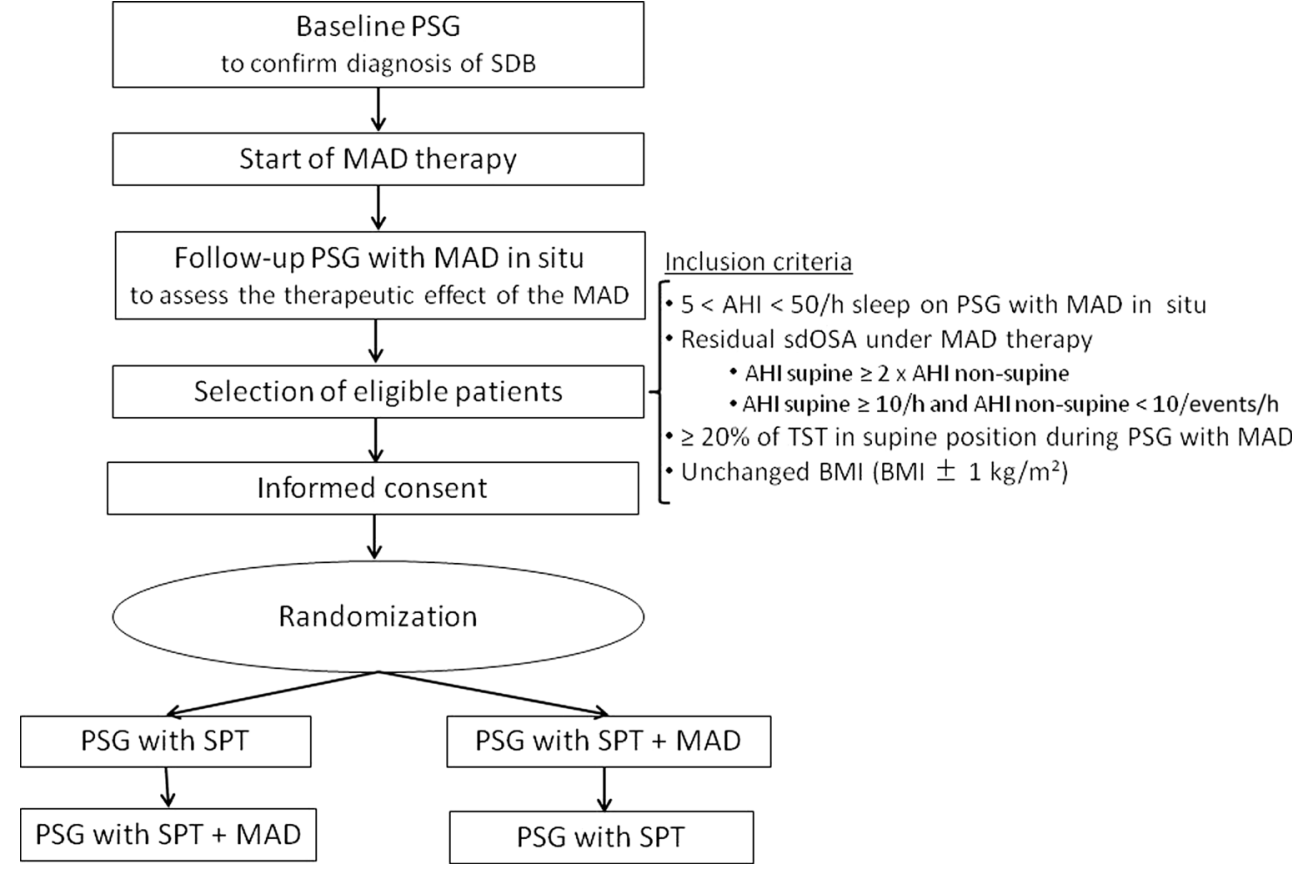

resolution of symptoms occurred or until the maximum comfortable limit was reached.

\section{Sleep position trainer}

A chest-worn SPT placed at the level of the sternum was used in this study (NightBalance ${ }^{\mathrm{TM}}$, Delft, The Netherlands) (Fig. 2) [14]. The SPT is a small, lightweight device $(72 \times$ $35 \times 10 \mathrm{~mm}, 25 \mathrm{~g})$ that allows for body position changes during sleep without any movement restrictions. The device continuously monitors position, providing a vibration stimulus when in supine position. The vibration is variable in frequency and gradually increases in amplitude and duration until the patient shifts to a non-supine position (Fig. 2).

Polysomnographic outcome measures

One individual who was blinded for the treatment assignment scored the polysomnographic evaluations. The severity of OSA was expressed by the overall AHI, defined as the average number of apneas and hypopneas per hour of sleep. The supine and non-supine AHIs were defined as the average number of apneas and hypopneas per hour of sleep occurring in the supine and non-supine position, respectively. The positional change index was defined as the total number of positional changes during sleep per hour of total sleep time [27]. Treatment response was defined as a reduction in overall AHI under therapy of $\geq 50 \%$ compared to baseline. Another success criterion used in this study was a reduction in overall AHI of $\geq 50 \%$ compared to baseline combined with a post-treatment AHI of $<5$ events $/ \mathrm{h}$.

Statistical analysis

Data were statistically analyzed using SPSS software (SPSS version 21, Statistical Package for Social Sciences, SPSS Inc., Chicago, Illinois, USA). A power calculation revealed that with 20 patients, a power of $80 \%$ with an effect size of 0.7 could be achieved at a significance level of $5 \%$.

The nonparametric Wilcoxon-signed rank test for paired observations was performed to compare different variables among the different study nights. Bonferroni correction for multiple comparisons was performed when comparing the results of the four different study nights. The significance
Fig. 2 The sleep position trainer used in this study

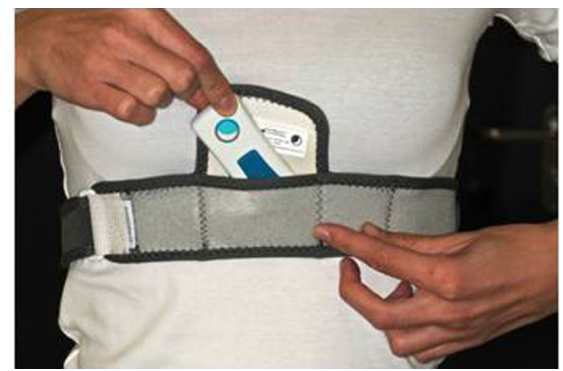


level was set at $p<0.05$, and the corrected significance level was $p<0.008$. The McNemar test was performed to compare the success rates of the different study nights. Since the data were not normally distributed, data are expressed as median values and their lower and upper quartiles $(\mathrm{Q} 1, \mathrm{Q} 3)$.

\section{Results}

\section{Subjects}

Twenty patients with sdOSA under MAD therapy (age, 52 \pm 11 years; male/female, 11/8; overall AHI, $24.6 \pm 10.2 / \mathrm{h}$; body mass index (BMI), $26.4 \pm 3.0 \mathrm{~kg} / \mathrm{m}^{2}$ ) were included in this RCT, see Table 1 for the baseline characteristics. There was no significant difference in age, weight, or sleep apnea severity between the two randomized groups. Four patients $(21 \%)$ were non-supine-dependent at baseline according to Cartwright's definition, but all of them became supinedependent under MAD therapy.

There was one patient who suffered from non-apneic snoring at baseline and was excluded from further analysis.

Time spent in supine sleeping position

Figure 3 shows the percentages of total sleep time spent in supine position during the four study nights: baseline, with MAD, with SPT, and with combination of SPT + MAD therapy. The SPT was found to be effective in reducing the time spent in supine position compared to baseline from $31.9(15.4 ; 52.4) \%$ at baseline to $0.0(0.0 ; 1.1) \%$ with SPT $(p=0.001)$.

In addition, the time spent in supine position was reduced with the SPT compared to MAD therapy, from 49.5 (32.6; $48.9) \%$ with MAD to $0.0(0.0 ; 1.1) \%$ with SPT $(p<0.001)$, respectively. The time spent in the supine position with SPT + MAD therapy was $0.0(0.0 ; 1.0) \%$ and was significantly lower compared to baseline $(p=0.001)$ or MAD therapy alone $(p<0.001)$.

Table 1 Baseline characteristics of the study population

\begin{tabular}{ll}
\hline Parameter & Mean \pm SD \\
\hline Age (years) & $52.5 \pm 10.5$ \\
Gender & $57.9 \%$ male \\
Body mass index, BMI $\left(\mathrm{kg} / \mathrm{m}^{2}\right)$ & $26.4 \pm 3.0$ \\
Overall apnea/hypopnea index, AHI (events/h) & $20.9(17.0 ; 34.0)$ \\
Supine AHI (events/h) & $39.1(26.4 ; 58.2)$ \\
Non-supine AHI (events/h) & $11.1(6.3 ; 26.1)$ \\
\hline
\end{tabular}

Data are expressed as mean \pm standard deviation (SD), percentages or median (Q1; Q3)

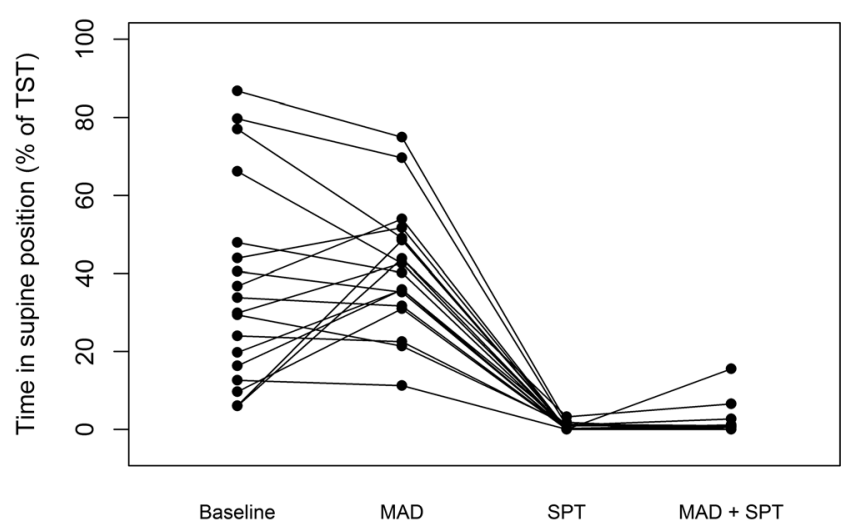

Fig. 3 Percentages of total sleep time spent (TST) in the supine position for the different study nights

Positional change index

The frequency of positional changes was similar between the different study nights. The positional change index at baseline was $2.0(1.6 ; 2.8) / \mathrm{h}$, which was not significantly different from the positional change index during MAD therapy $[1.7$ $(1.2 ; 2.4) / \mathrm{h}]$, SPT therapy $[2.2(1.6 ; 3.0) / \mathrm{h}]$, or combination therapy $[2.4(1.7 ; 3.4) / h]$.

Polysomnographic efficacy

The polysomnographic results of respiratory disturbances and sleep quality for the different study nights are shown in Table 2.

\section{Respiratory disturbances}

There was a significant difference in overall AHI between the four conditions tested, as graphically illustrated in Fig. 4. Both MAD and SPT were individually effective in reducing the median overall AHI significantly when compared to baseline, from $20.9(17.0 ; 34.0) / \mathrm{h}$ to $11.0(6.6 ; 14.0) / \mathrm{h}$ and to $21.8(3.9$; 17.9)/h, respectively. Combination SPT + MAD therapy further reduced the median overall AHI to $5.5(3.4 ; 7.2) / \mathrm{h}$. This was significantly lower when compared to baseline $(p<0.001)$, MAD alone $(p<0.001)$ and SPT alone $(p<0.008)$.

The supine AHI was reduced effectively with MAD when compared to baseline, from $39.1(26.4 ; 58.2) / \mathrm{h}$ to $21.8(14.8$; 29.5)/h. In addition, the SPT further reduced the supine AHI significantly when compared to MAD therapy to a supine AHI of $0.0(0.0 ; 11.5) / \mathrm{h}$.

In contrast to the SPT, MAD therapy was effective in reducing the non-supine $\mathrm{AHI}$ values when compared to baseline. Moreover, the non-supine AHI values with combination SPT + MAD therapy were significantly lower when compared to both baseline and SPT and comparable to that of the MAD alone. 
Table 2 Polysomnographic results of the different study nights

\begin{tabular}{|c|c|c|c|c|}
\hline Parameter & Baseline & With MAD & With SPT & With SPT + MAD \\
\hline Overall AHI (events/h) & $20.9(17.0 ; 34.0)$ & $11.0(6.6 ; 14.0)^{*}$ & $12.8(3.9 ; 17.9)^{*}$ & $5.5(3.4 ; 7.2)^{*, \$, \infty}$ \\
\hline Supine AHI (events/h) & $39.1(26.4 ; 58.2)$ & $21.8(14.8 ; 29.5)^{*}$ & $0.0(0.0 ; 11.5)^{*, \$}$ & $0.0(0.0 ; 22.7)^{*}$ \\
\hline Non-supine AHI (events/h) & $11.1(6.3 ; 26.1)$ & $3.9(2.0 ; 5.0)^{*}$ & $12.8(3.8 ; 17.2)^{\$}$ & $4.8(2.0 ; 6.0)^{*, \infty}$ \\
\hline ODI (events/h) & $7.7(6.6 ; 16.5)$ & $3.8(1.2 ; 5.5)^{*}$ & $2.6(1.0 ; 4.6)^{*}$ & $1.8(1.0 ; 3.0)^{*, \$}$ \\
\hline Mean $\mathrm{SaO}_{2}(\%)$ & $94.9(93.6 ; 95.8)$ & $94.8(93.8 ; 96.2)$ & $95.3(94.0 ; 96.2)$ & $95.4(94.1 ; 96.9)$ \\
\hline Min $\mathrm{SaO}_{2}(\%)$ & $84.7(79.0 ; 86.0)$ & $87.0(84.0 ; 91.0)$ & $88.0(84.0 ; 90.0)^{*}$ & $89.0(87.0 ; 91.0)^{*}$ \\
\hline TST (min) & $394.5(361.2 ; 420.6)$ & $419.0(407.0 ; 452.5)$ & $405.5(386.5 ; 438.2)$ & $413.0(385.0 ; 444.2)$ \\
\hline Sleep efficiency $(\%)$ & $85.1(79.4 ; 87.2)$ & $87.5(82.4 ; 92.6)$ & $84.6(80.6 ; 87.9)$ & $84.2(79.0 ; 89.7)$ \\
\hline Stage N1 (\% TST) & $5.8(3.4 ; 6.7)$ & $3.9(3.2 ; 5.6)$ & $4.4(3.3 ; 6.2)$ & $4.1(3.1 ; 4.9)$ \\
\hline Stage N2 (\% TST) & $51.4(38.7 ; 54.6)$ & $47.9(43.5 ; 51.0)$ & $48.7(48.0 ; 51.2)$ & $46.6(39.0 ; 50.2)$ \\
\hline Stage N3 (\% TST) & $11.9(5.9 ; 18.9)$ & $20.0(15.3 ; 22.8)^{*}$ & $15.2(12.3 ; 18.9)$ & $17.6(11.4 ; 22.7)$ \\
\hline Stage REM (\% TST) & $17.3(13.7 ; 21.3)$ & $21.2(15.3 ; 26.2)$ & $20.5(15.6 ; 26.1)$ & $20.5(19.1 ; 27.7)$ \\
\hline Arousal index (events/h) & $10.1(6.6 ; 15.0)$ & $9.2(7.3 ; 14.7)$ & $19.5(11.2 ; 24.3)^{\$}$ & $11.7(6.7 ; 13.6)^{\infty}$ \\
\hline Percentage of respiratory arousals (\%) & $35.4(24.8 ; 59.4)$ & $17.1(7.0 ; 32.0)^{*}$ & $19.6(4.7 ; 32.3)^{*}$ & $8.0(2.9 ; 12.9)^{* \$}$ \\
\hline Number of awakenings & $22.0(13.0 ; 29.0)$ & $17.0(13.3 ; 21.5)$ & $21.5(14.5 ; 30.0)$ & $18.0(13.3 ; 24.8)$ \\
\hline Percentage of TST in supine position (\%) & $31.9(15.4 ; 52.4)$ & $49.5(32.6 ; 48.9)$ & $0.0(0.0 ; 1.1)^{*, \$}$ & $0.0(0.0 ; 1.0)^{*, \$}$ \\
\hline Positional change index (changes/h) & $2.0(1.6 ; 2.8)$ & $1.7(1.2 ; 2.4)$ & $2.2(1.6 ; 3.0)$ & $2.4(1.7 ; 3.4)$ \\
\hline
\end{tabular}

Data are presented as median $(\mathrm{Q} 1, \mathrm{Q} 3)$

$A H I$ apnea/hypopnea index, $O D I$ oxygen desaturation index, TST total sleep time, REM rapid eye movement

* Statistically significant $(p<0.008)$ as compared to baseline

${ }^{\$}$ Statistically significant $(p<0.008)$ as compared to PSG with MAD

${ }^{\infty}$ Statistically significant $(p<0.008)$ as compared to PSG with SPT

\section{Sleep quality}

Total sleep time, sleep efficiency, and percentage of stage 1, stage 2, and REM sleep did not differ significantly between the different study nights (Table 2). There was a significant increase in stage 3 sleep with MAD compared to baseline. The arousal index increased significantly with SPT when compared to MAD therapy $(p<0.008)$ and combination SPT +
MAD therapy $(p<0.008)$ but not when compared to baseline. However, there was a decrease in the percentage of arousals associated with respiratory events during PSGs with MAD alone, SPT alone, and combination SPT + MAD therapy from $35.4(24.8 ; 59.4) \%$ at baseline to $17.1(7.0 ; 32.0) \%, 19.6(4.7$; $32.3) \%$, and $8.9(2.9 ; 12.9) \%$, respectively. The percentage of arousals associated with respiratory events was the lowest during combination SPT + MAD therapy.

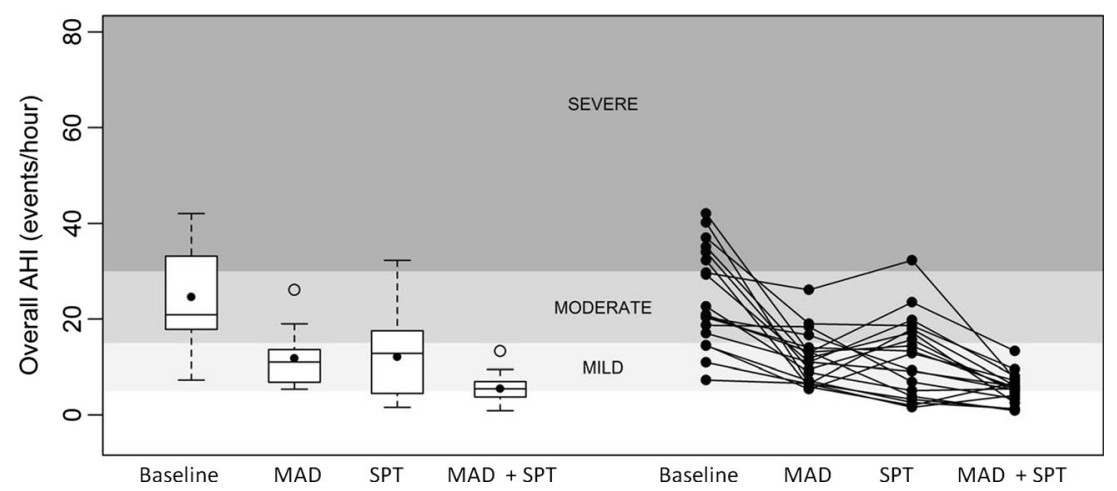

Fig. 4 Overall apnea/hypopnea index (AHI) for the different conditions tested. The different gray scales represent the varying levels of sleep apnea severity, ranging from normal nocturnal breathing (AHI $<5 / \mathrm{h}$ sleep), mild OSA (AHI 5-15/h), moderate OSA (AHI 15-30/h), to severe OSA (AHI $>30 / \mathrm{h}$ ). Left panel: Box plots for the four different study nights. The 75th and 25th percentiles are represented by the upper and lower margins, the mean values by the closed dots, and the median values by the horizontal line. Whiskers represent the maximum value (top) and the minimum value (bottom) of the dataset. Outliers are represented by an open dot. Right panel: The individual patient data for the different study nights are plotted in line graphs 


\section{Treatment outcome}

Overall, treatment response rate $(\geq 50 \%$ reduction in overall AHI when compared to baseline) was significantly higher with combination of SPT + MAD (95\%) when compared to MAD alone $(45 \% ; p<0.01)$ or SPT alone $(55 \% ; p<0.01)$. While using success defined as a reduction in overall AHI of $\geq 50 \%$ compared to baseline combined with a post-treatment AHI of $<5$ events $/ \mathrm{h}$, the success rates were 0,25 , and $35 \%$, respectively for MAD alone, SPT alone, or combination of SPT + MAD.

\section{Subjective preference of therapy}

Fifteen patients filled out the subjective questionnaire. Four patients identified MAD therapy as their subjective treatment of choice, four patients preferred SPT, and seven patients preferred the combination of SPT + MAD.

\section{Discussion}

This prospective RCT was the first to evaluate the feasibility and the efficacy of an additional chest-worn SPT in patients who were unsuccessfully treated with MAD therapy due to the presence of sdOSA under MAD therapy. The results indicate that the SPT used in this study significantly reduced the time spent in the supine position when compared to baseline or MAD therapy. Furthermore, both MAD and SPT were individually effective in reducing the apnea severity in patients with sdOSA under MAD therapy. In addition, combination SPT + MAD therapy leads to a higher therapeutic efficacy in patients with sdOSA under MAD therapy when compared to one of the treatment modalities alone.

It is reported in literature that a treatment modality for OSA that is not able to completely eliminate all breathing abnormalities leaves the patient with a residual OSA although often less severe than the initial OSA. Therefore, the residual OSA is probably more supine-dependent since supine-dependent OSA is more frequently seen in mild OSA. In a previous study, it was described that one third of patients under MAD therapy have a residual sdOSA. It was hypothesized that these patients with residual sdOSA under MAD therapy could benefit from additive therapy with SPT.

Most of the included patients ( $80 \%)$ were also supinedependent at baseline. In those patients, monotherapy with SPT should be considered as a first treatment option. However, in the past, most of the positional therapies had low acceptance and compliance due to the bulkiness of the devices and, therefore, was not frequently prescribed as a therapeutic option. Recently, the technology of positional therapy has been improved, with promising results.
Additionally, our results showed that the success rate of SPT alone was up to $55 \%$. The most promising results were achieved with combination SPT + MAD therapy, with success rates up to $95 \%$.

In the present study, $95 \%$ of patients were responders to combination SPT + MAD therapy based on success criteria defined as a reduction in overall $\mathrm{AHI}$ of $\geq 50 \%$ according to literature [28]. MAD therapy was effective in reducing both supine AHI and non-supine AHI when compared to baseline (Table 2). This indicates that MAD therapy, aimed at increasing the cross-sectional upper airway volume by advancing the mandible, has an effect on both non-supine AHI and supine AHI. On the other hand, the mode of action of the SPT is preventing the patients from the supine sleeping position. The results show that the time spent in supine position decreased dramatically with the SPT. In some patients, the AHI supine remained still high showing that these patients still have a problem when sleeping in the supine position, but with reducing the time spent in the supine position, the sleep apnea severity decreased in those patients.

The arousals caused by the SPT alone were significantly higher than those by the MAD alone or combination therapy. In addition, the percentage of arousals associated with respiratory events decreased during the study nights with MAD therapy, SPT, and combination SPT + MAD therapy. This indicates that the SPT is associated with an increase in arousals when compared to baseline or MAD therapy, especially those arousals not associated with respiratory events were increased. In future studies, synchronization of the timeline of the SPT with that of the PSG could be of additional interest in order to determine whether or not the arousals are associated with the vibration alarm of the SPT that forces the patient to change body position. In two other studies, one short term (28 days) [14] and one long term (6 months) [29], patients followed a training program to get used to the SPT after which no differences in arousal index were found between baseline and with SPT.

To date, only one paper has been published comparing the efficacy of an oral appliance and positional therapy [5]. In that study, Cartwright et al. [5] described the efficacy of combination therapy of a posture alarm giving an auditory beep when in supine position and a tongue retaining device (TRD), an oral appliance that holds the tongue in a forward position. Patients were assigned to either therapy with the posture alarm, the TRD, or combination therapy with the posture alarm and the TRD. The results of the present RCT are in accordance with the results of Cartwright et al. suggesting that the combination of an oral appliance and positional therapy is better than one of the treatment modalities alone.

Recently, a new classification system for sdOSA was proposed, the Amsterdam Positional OSA Classification (APOC), to classify patients in either true sdOSA patients who could be cured by PT alone or in patients who showed 
benefit from PT but could not be cured and therefore would benefit from combination of therapies [30].

The present study has its limitations. First, a relatively small number of patients was included in this prospective RCT. However, the present study was a proof of concept to evaluate the possible application and first results of this specific combination therapy. Second, the present study did not incorporate an SPT training program, allowing the patients to gradually get used to avoid the supine sleeping position [14]. Third, the MAD was titrated upon subjective resolution of symptoms or until reaching the maximum comfortable limit. The patients selected for this study were treated unsuccessfully and could have needed additional mandibular advancement. However, there was a clear residual sdOSA under MAD therapy. These patients could therefore benefit from additional PT in combination with the MAD instead of further titrating the MAD. Further titration of the MAD includes a risk of more side-effects due to the more advanced mandible. Finally, compliance was not taken into account since the patients were using the SPT the whole night. However, the SPT used in this study includes a feature for measuring compliance objectively, showing a compliance rate at 6-month follow-up of $64.4 \%$ in another study [29].

Despite the limitations, the authors stress the clinical relevance of the results of this prospective RCT. In the past, it has been shown that up to one third of patients treated with MAD therapy have sdOSA under therapy [23]. The results of the present study indicate that a combination of SPT + MAD therapy further reduces the sleep apnea severity when compared to the individual treatment modalities. In addition, the success rate with combination therapy increased by up to $65 \%$ points when compared to MAD therapy alone. These findings suggest that when patients are unsuccessfully treated with MAD therapy, the presence of sdOSA should be checked and combination SPT + MAD therapy could be suggested in eligible patients. However, further clinical trials are required to assess the applicability, the therapeutic efficacy, and compliance rates at long-term follow-up with this combination therapy, and with other possible combination therapies such as SPT + CPAP therapy.

\section{Conclusions}

The results of this prospective randomized controlled trial indicate that the SPT used in this study significantly reduced the time spent in the supine position compared to baseline or MAD therapy. In patients with sdOSA under MAD therapy, both MAD therapy and SPT were individually effective in reducing AHI. However, combination SPT + MAD therapy further reduced the sleep apnea severity and leads to a significantly higher OSA alleviation in patients with sdOSA under MAD therapy.

\section{References}

1. Cartwright RD (1984) Effect of sleep position on sleep apnea severity. Sleep 7(2):110-114

2. Mador MJ, Kufel TJ, Magalang UJ, Rajesh SK, Watwe V, Grant BJ (2005) Prevalence of positional sleep apnea in patients undergoing polysomnography. Chest 128(4):2130-2137

3. Marklund M, Persson M, Franklin KA (1998) Treatment success with a mandibular advancement device is related to supinedependent sleep apnea. Chest 114(6):1630-1635

4. Marklund M, Stenlund H, Franklin KA (2004) Mandibular advancement devices in 630 men and women with obstructive sleep apnea and snoring: tolerability and predictors of treatment success. Chest 125(4):1270-1278

5. Cartwright R, Ristanovic R, Diaz F, Caldarelli D, Alder G (1991) A comparative study of treatments for positional sleep apnea. Sleep 14(6):546-552

6. Safiruddin F, Koutsourelakis I, de Vries N (2014) Analysis of the influence of head rotation during drug-induced sleep endoscopy in obstructive sleep apnea. Laryngoscope. doi:10.1002/lary-24598

7. De Vito A, Carrasco Llatas M, Vanni A, Bosi M, Braghiroli A, Campanini A, de Vries N, Hamans E, Hohenhorst W, Kotecha BT, Maurer J, Montevecchi F, Piccin O, Sorrenti G, Vanderveken OM, Vicini C (2014) European position paper on drug-induced sedation endoscopy (DISE). Sleep Breath. doi:10.1007/s11325-014-0989-6

8. Oksenberg A, Silverberg D, Offenbach D, Arons E (2006) Positional therapy for obstructive sleep apnea patients: a 6-month follow-up study. Laryngoscope 116(11):1995-2000

9. Bignold JJ, Deans-Costi G, Goldsworthy MR, Robertson CA, McEvoy D, Catcheside PG, Mercer JD (2009) Poor longterm patient compliance with the tennis ball technique for treating positional obstructive sleep apnea. J Clin Sleep Med 5(5):428-430

10. Jokic R, Klimaszewski A, Crossley M, Sridhar G, Fitzpatrick MF (1999) Positional treatment vs continuous positive airway pressure in patients with positional obstructive sleep apnea syndrome. Chest 115(3):771-781

11. Permut I, Diaz-Abad M, Chatila W, Crocetti J, Gaughan JP, D'Alonzo GE, Krachman SL (2010) Comparison of positional therapy to CPAP in patients with positional obstructive sleep apnea. J Clin Sleep Med 6(3):238-243

12. Loord H, Hultcrantz E (2007) Positioner - a method for preventing sleep apnea. Acta Otolaryngol 127(8):861-868

13. Ravesloot MJ, van Maanen JP, Dun L, de Vries N (2013) The undervalued potential of positional therapy in position-dependent snoring and obstructive sleep apnea-a review of the literature. Sleep Breath 17(1):39-49

14. van Maanen JP, Meester KA, Dun LN, Koutsourelakis I, Witte BI, Laman DM, Hilgevoord AA, de Vries N (2013) The sleep position trainer: a new treatment for positional obstructive sleep apnoea. Sleep Breath 17(2):771-779

15. Bignold JJ, Mercer JD, Antic NA, McEvoy RD, Catcheside P (2011) Accurate position monitoring and improved supine-dependent obstructive sleep apnea with a new position recording and supine avoidance device. J Clin Sleep Med 7(4):376-383

16. Kushida CA, Littner MR, Hirshkowitz M, Morgenthaler TI, Alessi CA, Bailey D, Boehlecke B, Brown TM, Coleman J Jr, Friedman L, Kapen S, Kapur VK, Kramer M, Lee-Chiong T, Owens J, Pancer JP, Swick TJ, Wise MS (2006) Practice parameters for the use of continuous and bilevel positive airway pressure devices to treat adult patients with sleep-related breathing disorders. Sleep 29(3):375-380

17. Chan AS, Sutherland K, Schwab RJ, Zeng B, Petocz P, Lee RW, Darendeliler MA, Cistulli PA (2010) The effect of mandibular 
advancement on upper airway structure in obstructive sleep apnoea. Thorax 65(8):726-732

18. Ryan CF, Love LL, Peat D, Fleetham JA, Lowe AA (1999) Mandibular advancement oral appliance therapy for obstructive sleep apnoea: effect on awake calibre of the velopharynx. Thorax 54(11): 972-977

19. Sutherland K, Vanderveken OM, Tsuda H, Marklund M, Gagnadoux F, Kushida CA, Cistulli PA (2014) Oral appliance treatment for obstructive sleep apnea: an update. J Clin Sleep Med 10(2):215-227

20. Hoffstein V (2007) Review of oral appliances for treatment of sleepdisordered breathing. Sleep Breath 11(1):1-22

21. Vanderveken OM, Dieltjens M, Wouters K, De Backer WA, Van de Heyning PH, Braem MJ (2013) Objective measurement of compliance during oral appliance therapy for sleep-disordered breathing. Thorax 68(1):91-96

22. Dieltjens M, Braem MJ, Vroegop AV, Wouters K, Verbraecken JA, De Backer WA, Van de Heyning PH, Vanderveken OM (2013) Objectively measured vs self-reported compliance during oral appliance therapy for sleep-disordered breathing. Chest 144(5):14951502

23. Dieltjens M, Braem MJ, Van de Heyning PH, Wouters K, Vanderveken OM (2014) Prevalence and clinical significance of supine-dependent obstructive sleep apnea in patients using oral appliance therapy. J Clin Sleep Med 10(9):959-964

24. Ten Berge DM, Braem MJ, Altenburg A, Dieltjens M, Van de Heyning PH, Vanhaecht K, Vanderveken OM (2014) Evaluation of the impact of a clinical pathway on the organization of a multidisciplinary dental sleep clinic. Sleep Breath 18:325-334

25. Vanderveken OM, Devolder A, Marklund M, Boudewyns AN, Braem MJ, Okkerse W, Verbraeceken JV, Franklin KA, De Backer WA, Van de Heyning PH (2008) Comparison of a custom-made and a thermoplastic oral appliance for the treatment of mild sleep apnea. Am J Respir Crit Care Med 178(2):197-202

26. Petit FX, Pepin JL, Bettega G, Sadek H, Raphael B, Levy P (2002) Mandibular advancement devices: rate of contraindications in 100 consecutive obstructive sleep apnea patients. Am J Respir Crit Care Med 166(3):274-278

27. Choi JH, Kim EJ, Choi J, Kwon SY, Lee HM, Kim TH, Lee SH, Shin C, Lee SH (2011) Effect of successful surgical treatment on changes of position during sleep in adults with obstructive sleep apnea syndrome. Ann Otol Rhinol Laryngol 120(2):104-109

28. Ferguson KA, Cartwright R, Rogers R, Schmidt-Nowara W (2006) Oral appliances for snoring and obstructive sleep apnea: a review. Sleep 29(2):244-262

29. Van Maanen P, de Vries N (2014) Long-term effectiveness and compliane of positional therapy with the sleep position trainer in the treatment of positional obstructive sleep apnea syndrome. Sleep 37(7):1209-1215

30. Frank MH, Ravesloot MJ, van Maanen JP, Verhagen E, de Lange J, de Vries N (2014) Positional OSA part 1: towards a clinical classification system for position-dependent obstructive sleep apnoea. Sleep Breath. doi:10.1007/S11325-014-1022-9 